\title{
Quitting: In male versus female users of different types of tobacco at 8 months postintervention in a community setting
}

\section{Srabana Misra Bhagabaty, Amal Chandra Kataki', Manoj Kalita ${ }^{2}$, Shekhar Salkar ${ }^{3}$}

Department of Preventive Oncology, ${ }^{1}$ Director, and ${ }^{2}$ Department of Epidemiology and Biostatistics, Dr. B. Borooah

Cancer Institute, Guwahati,

Assam, ${ }^{3}$ Department of ENT, National Organization for Tobacco Eradication, Goa, India

Address for the Correspondence:

Dr. Srabana Misra Bhagabaty, Department of Preventive Oncology, Dr. B. Borooah Cancer Institute, Guwahati, Assam, India. E-mail: srabana.misra@gmail.com

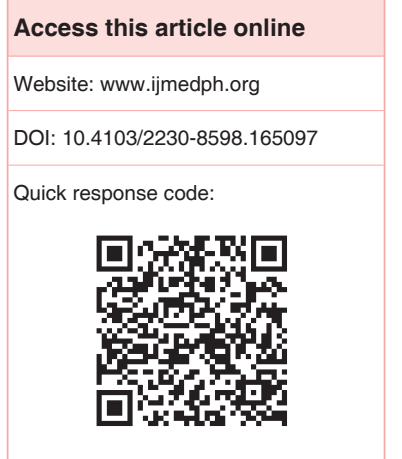

Background: North-East India shows a very high prevalence of tobacco use. Very little is known about the factors that affects tobacco cessation. Materials and Methods: Community-based tobacco cessation counseling was provided and follow-up visits were done until 8 months. Retrospective analysis was done on data set collected during the period of 2009-2010. Both sexes of age $>15$ years with mild, moderate and severe category of tobacco addiction and giving consent for the intervention were included in the study. Descriptive statistics and Chi-square test were used to see the significance differences among categories. Results: Totally, 800 tobacco users were intervened and followed-up. Male:female ratio of tobacco use was 4.3:1. Against one female, 27 male smokers were found. At 8 months postintervention, maximum number of quit rate was amongst the users of smokeless tobacco (52\%) irrespective of their sex as well as in both sexes. The difference in quit rates between female smokeless tobacco users and female smokers was found statistically significant. No female smokers quitted tobacco whereas $46.6 \%$ male smokers quitted tobacco. Conclusion: Quit rate in females with different types of tobacco use habits differ significantly. There is an alarming disparity in the quit rates of male versus female smokers which needs further research. Capacity development of the cessation service providers toward the liberal use of nicotine replacement therapy and other chemo interventions in addition to counseling should be further facilitated.

Key words: North-East India, quitting, rates, tobacco

\section{INTRODUCTION}

Tobacco is a well-known preventable cause of death worldwide. According to World Health Organization, tobacco kills nearly 6 million people every year and it is one of the biggest epidemic world has ever faced. More than 5 million deaths are due to direct use of tobacco while more than 600,000 are the result of nonsmokers being exposed to second-hand smoke. ${ }^{[1]}$ India is the second largest consumer and third largest producer of tobacco. ${ }^{[2]}$ Nearly, 900,000 people die every year in India due to diseases attributed to tobacco. ${ }^{[3]}$ Tobacco is used in various forms worldwide. In India, tobacco is used in the form of smoked, chewed, and even consumed as drink like in Mizoram of North-East India a unique form of water extract of tobacco smoke called tuibur is used by people. North-East India shows a very high prevalence of tobacco use. Cultural acceptance makes the tobacco use habit more prevalent in this part of the country. More than $50 \%$ of cancer cases to all cancer cases are tobacco related in these regions of North-East India and observed women are at most risk. In most of the places in North-East India, it is common practice among women to smoke and chew tobacco related substances. ${ }^{[4]}$ Global Adult Tobacco Survey 2009-2010 shows that 34.6\% adults in India are current tobacco users out of which $8.7 \%$ are smokers, $20.6 \%$ are users of smokeless tobacco and $5.3 \%$ are current users of both smoking and smokeless forms. ${ }^{[3]}$ In Assam, 39.3\% adults are current tobacco users out of which $6.6 \%$ smoke, $24.9 \%$ use smokeless and $7.8 \%$ use in both smoking and smokeless forms. Among males in India, 15\% only smoke, $24 \%$ use only smokeless tobacco and $9 \%$ use tobacco in both forms. About $2 \%$ females in India only smoke, $17 \%$ use only smokeless tobacco and 1\% uses both forms. Quitting and maintenance of the action phase of tobacco cessation is a challenge for both the user and the cessation service provider. In North-East India maximum tobacco cessation 
intervention being through counseling only which is $27 \%{ }^{[5]}$ In our clinical setting of Regional Cancer Center, we observed many cancer patients could not quit tobacco in spite of all efforts on patients part and special cessation helps provided to the patients. Here, in this study, we tried to analyze if there is any difference in quit rate depending on the type of tobacco use and sex of the user and if there is any scope of improving and maintaining quit rate amongst the tobacco users.

\section{Objectives}

1. To analyze if there is any difference in quit rates depending on the different type of tobacco users at 8 months postintervention at a community setting.

2. To speculate the if the quit rate in female tobacco users of varying patterns tally with their male counterparts.

3. To consider specific intervention procedures toward better quit rate depending on the type of use and user.

\section{MATERIALS AND METHODS}

\section{Data source and methodology}

Data were obtained from a community-based tobacco cessation intervention project in the year 2009-2010. Duration of this intervention design was 12 months. The study sample was 800 tobacco users who gave consent to be included in the study. The inclusion criteria for the study subjects were, both male and females of age $>15$ years with mild, moderate and severe category of tobacco addiction, where smoking 20 or more cigarettes or biddies per day or using other forms of tobacco 10 or more times a day was considered as severe, 10-19 cigarettes/biddies/day or 5-9 times other tobacco use as moderate and $<10$ cigarettes/biddies/day or using other forms of tobacco $<5$ times/day as mild category of tobacco addiction.

The exclusion criteria were nonusers of tobacco, tobacco users of age $<15$ years, not giving consent for intervention, not permanent residents of the study areas.

It was carried out in four that is, South, East, West and Central zones of Guwahati metro of Assam. The first 2 months were used for door to door survey in the intervention areas to find out the intervention sample. Four medical social workers (MSW) carried out the community-based intervention and follow-up. Each of the MSW was allotted with one zone. The MSWs were trained up on the tobacco scenario, tobacco hazards and counseling and basic knowledge on tobacco control and cessation services.

In our study, we used only counseling as a tool of intervention for tobacco cessation.

These MSW surveyed their respective areas door to door starting from one end of the areas with a prominent landmark to find out the find out the sample for intervention which was followed by registration of the study subjects till they found the required number of study subjects. A predesigned proforma was filled up during registration of the study subjects. A detailed history of the tobacco use pattern along with addiction and motivation level was included in the proforma. Proforma contained follow-up status estimation questions were filled up during each follow-up.

During registration, information education and communications materials on tobacco hazards were offered to the subjects and sensitized them on the tobacco hazards as well as date and time for counseling was fixed according to the convenience of the subjects as well as keeping in mind time frame of the project. The intervention was tobacco cessation counseling offered by the MSW at the community setting at homes of the study subjects. After counseling, the subjects were followed-up for 8 months. Follow-up was done by direct contact at 2 weeks, 4 weeks, 6 weeks, 2 months, 3 months, 4 months, 6 months, 8 months. When contacted for follow-up if needed re counseling was given to the study subjects.

The results of the present study are presented as percentages up to single decimal place. Descriptive statistics was calculated, and Chisquare test was assessed to test the significance differences among categories; $P<0.05$ was considered as statistically significant at $95 \%$ confidence interval.

\section{RESULTS}

Seven hundred and fifty households were surveyed and 2250 adults of $>15$ years age were found out of which 809 were tobacco users. A total of 800 total study subjects fulfilling inclusion criteria were registered. Of the 9 excluded subjects, all were males and 7 were tobacco chewers and 2 were smokers. From here, it becomes obvious that in the study area, $36 \%$ adults were tobacco users. Irrespective of sex, smokeless tobacco use addiction is around 14\%. About $12 \%$ of the sample used in both smoking and smokeless form and $10 \%$ only smoked.

Sex distribution of tobacco users showed 649 were male users and 151 were female users of tobacco in any form. About 33\% males only smoked and $42 \%$ used tobacco in both forms and $25.4 \%$ male users used smokeless tobacco. Of $25 \%$ males, users of smokeless tobacco and majority used it in khaini form (16\%).

About $94.6 \%$ female users used smokeless forms of tobacco. Of these, $94.6 \%$ females, $51 \%$ used betal quid with raw tobacco leaf and lime, $21 \%$ used khaini, $4 \%$ chewed zarda pan, $16 \%$ chewed guthkha and $2 \%$ used multiple smokeless products.

Distribution of quitters according to their tobacco use habit irrespective of sex is shown in Table 1. Chi-square test for the difference of type of use and quitting tobacco showed Chi-square $=3.036$, degree of freedom was 2 with $P=0.2191$.

In Table 2, distribution of male quitters according to the type of tobacco use habit is highlighted. The association between different tobacco use pattern and quit rate amongst males gave Chi-square $=2.123$, degree of freedom $2, P>0.05$. 
The significance of difference between female quitters of tobacco according to the type of use was tested by Chi-square test [Table 3]. On comparing between different use type and quit rate, it was found as statistically significant with value of Chi-square statistic 4.311, at degree of freedom 2 and $P=0.0379$.

\section{DISCUSSION}

In the study sample, $36 \%$ adults above 15 years were current tobacco users. This percentage tallies with the national level that is $34.6 \%$. But Assam shows a little higher rate of $39.3 \%{ }^{[3]}$ The majority of the users had only smokeless tobacco use habit that is around $14 \%$. Next category of use is in both smoking and smokeless form $(12 \%)$ and then smoking form $(10 \%)$. In India, $8.7 \%$ are smokers, $20.6 \%$ are users of smokeless tobacco and $5.3 \%$ are current users of both smoking and smokeless forms irrespective of sex and these figures in Assam are $6.6 \%, 24.9 \%$ and $7.8 \%$ respectively. ${ }^{[3]}$ Here, it became obvious that number of smokers as well as users of both smokeless and smoking forms in the study sample is higher than national and state level.

Irrespective of sex, in these study areas smokeless form of tobacco use was found to be more prevalent in comparison to the smoking form.

In the study sample male:female ratio of tobacco use was 4.3:1, which is higher than the national scenario where tobacco use in males is around two-folds higher than female. The majority of males used in tobacco in both forms whereas females mainly used tobacco in smokeless form. When we compared the pattern between males and females we found that the male:female ratio of smoking form of tobacco use is around 27:1. About smokeless form, it became obvious from intra group comparison that female chew more and

$\begin{aligned} & \text { Table 1: Distribution of tobacco quitters according } \\
& \text { to the type of use }\end{aligned}$
\begin{tabular}{lccc}
\hline Habits & Users & Quitters & Percentage of quit \\
\hline Smoking habit only & 221 & 103 & 46.6 \\
Smokeless tobacco habit only & 308 & 162 & 52 \\
Smoking and smokeless habit & 271 & 110 & 40.59 \\
\hline
\end{tabular}

\begin{tabular}{|c|c|c|c|}
\hline Habits & Users & Quitters & Percentage of quit \\
\hline Smoking habit only & 213 & 103 & 48.36 \\
\hline Smokeless tobacco habit only & 165 & 85 & 51.52 \\
\hline Smoking and smokeless habit & 271 & 110 & 40.59 \\
\hline
\end{tabular}

$\begin{aligned} & \text { Table 3: Distribution of female quitters with different } \\
& \text { tobacco use habit }\end{aligned}$
\begin{tabular}{lcccc} 
Habits & Users & Quitters & Percentage of quit \\
\hline Smoking habit only & 8 & 0 & 0 \\
Smokeless tobacco habit only & 142 & 78 & 54.9 \\
Smoking and smokeless habit & 0 & 0 & 0 \\
\hline
\end{tabular}

males smoke more. About $25.4 \%$ male users used smokeless tobacco whereas $94.6 \%$ female users were into the habit of using smokeless forms of tobacco. Maximum no of female chewers gave history of use of betel quid where raw/processed areca nut, betel leaf along with lime and raw tobacco leaf were used. Out of male users of smokeless tobacco and majority used it in khaini form.

Nearly half (47\%) of current smokers and smokeless tobacco users $(46 \%)$ want to quit tobacco and $38 \%$ made an attempt to quit tobacco. About 26.7\% male smokers and 19.6\% female smokers almost were quitters at 1-4 years since quitting which shows less difference at $10+$ years which is $49 \%$ in males and $43.8 \%$ in females. Though there is less difference, still female smokers shows lower quit rate in comparison to their male counterpart. In case of smokeless tobacco users, almost equal numbers of male $(25 \%)$ and female $(27 \%)$ users of smokeless tobacco were quitters at $1-4$ years since quitting which $26.4 \%$ in males and $24.5 \%$ at $10+$ years postquitting. ${ }^{[2]}$

When we analyzed the quit rates amongst the users of different types of tobacco at 8 months postintervention irrespective of sex difference, interesting finding observed is that maximum number of quit rate was amongst the users of smokeless tobacco (52\%). Those who were using both smoking and smokeless forms of tobacco showed the least quit rate $(40.59 \%)$. The smokers showed quit rate in between these two groups (46.6\%).

Though the difference in quit rate between different types of tobacco users irrespective of sex is was not statistically significant.

When we considered only male users and analyzed quit rates amongst different types of tobacco users amongst them, it was obvious that in male users of both forms of tobacco quitted less $(40.59 \%)$ whereas those who only used smokeless forms gave a higher quit rate $(51.52 \%)$. Smokers showed results in between these two groups $(48.36 \%)$. The difference of quit rates depending on the type of use being insignificant in male user when it was analyzed by Chi-square test. The difference in quit rates among different types of tobacco users was found insignificant in males.

Maximum number of female tobacco used tobacco in smokeless forms which is $94 \%$ of the female tobacco users. Astonishing fact we noticed during our analysis was that no female smokers quitted the habit at 8 months postintervention follow-up. Quit rate was zero for female smokers. However, $54.9 \%$ female chewers quitted successfully and were found in the maintenance phase at 8 months postintervention follow-up. The difference in quit rates between female smokeless tobacco users and female smokers was found significant.

When it comes to only smokeless tobacco users, quit rate slightly differed amongst male and female that is, $51.5 \%$ male and a slightly higher percentage of females $(54.9 \%)$ quitted tobacco as recorded at 8 months postintervention follow-up.

In our study, of 221 smokers, majority were males that accounted for $96.4 \%$, whereas only $4 \%$ were female smokers. But astonishing 
fact that has been observed was that no female smokers quitted tobacco whereas $46.6 \%$ male smokers quitted the habit as recorded at 8 months visit postintervention follow-up at community setting. We tried to compare the difference of quit rate in male versus female smokers. This is a question raising situation that is female smokers are more addicted in comparision to their male counterparts ... is it necessary to add nicotine replacement therapy (NRT) with or without use of antidepressants in female smokers along with behavior change counseling sessions needs more research. Smoking prevalence is lower among women than men in most countries. Approximately, $22 \%$ of women in developed countries and $9 \%$ of women in developing countries smoke, but because most women live in developing countries, there are numerically more women smokers in developing countries. Unless effective, comprehensive and sustained initiatives are implemented to reduce smoking uptake among young women and increase cessation rates among women, the prevalence of female smoking in developed and developing countries is likely to rise to $20 \%$ by $2025 .{ }^{[6]}$ But comparison between quitting status in male versus female smokers showed nearing half of the male smokers quitted and were found in maintenance phase at 8 months postintervention but no female smokers quitted even after repeated counseling and regular follow-up visits. The percentage difference between male versus female smokers itself is a story taller. Women are less successful than men at quitting smoking. ${ }^{[7]}$ The lifetime quit rate (number of ex-smokers/number of ever smokers) $\times 100$ was higher in males than in females. ${ }^{[8]}$ Not only female smokers quit less than female chewers in our research significantly but also female smokers alarmingly shoulder high quitting challenges for the cessation service provider than the male smoker counterpart.

\section{CONCLUSION AND RECOMMENDATION}

Males were found to use both smoking and smokeless forms of tobacco predominantly, and females were predominant users of smokeless forms. The majority of the women used areca and betal leaf with lime and raw tobacco. Quit rates as such did not differ significantly based upon the type of tobacco use irrespective of sex of the user. No such quit rate difference had been observed in intra group comparison between male users of different types of tobacco. In case of female, quit rate for smokers was zero and the difference of quit rates amongst smokers and smokeless tobacco users was statistically found to be significant. The quit rate difference between male and female smokers was quite alarming. To know more about this disparity, more research is needed in this field. In this intervention study, we used only counseling as an intervention tool. For better quit results among users of tobacco, capacity development of the tobacco cessation service providers, toward the use of NRT or antidepressants should be given more emphasis on. Counseling for cessation at both clinic-based and community settings should be added by liberal use of NRT and chemo interventions for more challenging patterns of tobacco use for example those who uses both that is, smoking and smokeless forms and female smokers.

\section{REFERENCES}

1. World Health Organization. Available from: http://www.who.int/ mediacentre/factsheets/fs339/en. [Last accessed on 2015 Feb 10].

2. Jhanjee S. Tobacco control in India - Where are we now? Delhi Psychiatry J 2011;14:26-32.

3. International Institute for Population Sciences (IIPS), Mumbai and Ministry of Health and Family Welfare, Government of India: Global Adult Tobacco Survey India (GATS India), 2009-2010. New Delhi; 2010.

4. Sharma JD, Kalit M, Nirmolia T, Saikia SP, Sharma A, Barman D. Cancer: Scenario and relationship of different geographical areas of the globe with special reference to North East-India. Asian Pac J Cancer Prev 2014;15:3721-9.

5. Jayakrishnan R, Mathew A, Uutela A, Finne P. A community based smoking cessation intervention trial for rural Kerala, India. Asian Pac J Cancer Prev 2011;12:3191-5.

6. Mackay J, Amos A. Women and tobacco. Respirology 2003;8:123-30.

7. Kabat GC, Wynder EL. Determinants of quitting smoking. Am J Public Health 1987;77:1301-5.

8. Bohadana A, Nilsson F, Rasmussen T, Martinet Y. Gender differences in quit rates following smoking cessation with combination nicotine therapy: Influence of baseline smoking behavior. Nicotine Tob Res 2003;5:111-6.

How to cite this article: Bhagabaty SM, Kataki AC, Kalita M, Salkar $S$. Quitting: In male versus female users of different types of tobacco at 8 months postintervention in a community setting. Int J Med Public Health 2015;5:353-6.

Source of Support: Nil, Conflicts of Interest: None declared. 